\title{
Las lagunas en el Derecho y la interpretación constitucional*
}

\section{Gaps in the Law and Constitutional Interpretation}

Fecha de recepción: 13 de junio de 2009

Fecha de aprobación: 23 de septiembre de 2009

Feridnand Melin -Soucramanien**

Una medida del verdadero espíritu de lo clásico implica necesariamente de silencios. Igualmente, en la pintura, los maestros holandeses demostraron con empeño que el papel de la sombra era precisamente hacer resaltar la luz con mayor intensidad.

En el Derecho ocurre algo parecido. Son las lagunas las que dibujan los contornos del orden jurídico, porque ellas permiten dar una imagen, "de manera indirecta", del sistema normativo. Si se entiende bien el término de laguna no puede ser utilizado en Derecho más como una metáfora. Etimológicamente, laguna (lacuna en latín), define a un pequeño lago, un repliegue de un terreno; por extensión, el término hace alusión a un espacio vacío. En Derecho cuando hablamos de laguna, nos referimos en sentido estricto a un estado inacabado-imperfecto (de no plenitud) del aparato normativo. Esta primera definición, que parece neutra en apariencia, descansa sobre el postulado de que puede existir un orden jurídico completo, un orden jurídico perfecto.

Pero, también existe una segunda definición del término que es, de hecho, la más utilizada. Es aquélla según la cual las lagunas muestran una falta o una deficiencia cualquiera, lo cual afecta la solución jurídica de un problema particular. Tomado en este sentido, el término de laguna comporta una connotación necesariamente negativa. Por otra parte, así como estas connotaciones muestran una brecha dentro de la unidad del Derecho o una insuficiencia en la solución jurídica, las lagunas son generalmente presentadas como anomalías. Ellas reclaman remedios (o acciones) apropiadas. Se tratará entonces de "subsanar las lagunas", es

El presente texto corresponde a la conferencia dictada por dicho profesor como docente invitado a la Maestría de Derecho Público de la Universidad Santo Tomás, en el marco del convenio USTA - Montesquieu - Bordeaux IV.

** Profesor - investigador de la Universidad de Montesquieu - Bordeaux IV (Francia). 
decir, de llenar los espacios vacíos existentes en las normas del Derecho positivo.

Nos referiremos aquí al tema de las lagunas en el Derecho y, particularmente, en el Derecho Constitucional. El Derecho, entendido como un sistema normativo y el Derecho Constitucional, en cuanto tal como la matriz de ese sistema normativo, en la medida en que la Constitución representa, como lo demostró Kelsen, "una norma de producción de normas". Pero, aun reducido el problema a este aspecto, el tema de las lagunas parece todavía extremadamente amplio. Para delimitarlo más aún, no resulta inútil recordar el contexto histórico en el cual esta cuestión comenzó a ser considerada por la doctrina.

En Europa, podemos remontarnos a las primeras inquietudes sobre este punto desde el Antiguo Régimen, a partir del siglo XIII, con el abandono de una concepción irracional del Derecho en provecho de soluciones más racionales. Sobre todo, con el cambio profundo que opera a lo largo del siglo XVI, cuando se impone la idea de una superioridad del Derecho escrito. A partir de este instante, el Derecho puede ser visto como un sistema de normas y, lógicamente, el tema de las lagunas en dicho sistema se puede comenzar a plantear.

Pero, evidentemente en esta época, en Francia, la cuestión es rápidamente resuelta, porque prevalecía el principio del absolutismo monárquico. En efecto, es al Rey, fuente de toda justicia y legislador soberano, a quien en última instancia le compete subsanar las eventuales lagunas. Desde ese punto de vista, la Revolución Francesa representa una ruptura decisiva con el afianzamiento del principio de la separación de las funciones jurisdiccionales y legislativas, en la Ley del 16-24 de agosto de 1790.

En la dirección de las reflexiones consignadas por Montesquieu en el Espíritu de las leyes, la distribución de roles entre los órganos, aparece evidentemente clara. Al legislador le corresponde la función de "fabricar" la ley y al juez la de aplicarla mediante un simple análisis silogístico. En esta definición mecánica, los tribunales no pueden, como en el Antiguo Régimen, crear el Derecho a través de la interpretación de una ley. Si una laguna aparece, corresponde solamente al legislador, por vía de un recurso de urgencia legislativa, subsanarla.

Esta nueva perspectiva vivió su apogeo durante el siglo XIX con el movimiento codificador, bien conocido, con la Escuela de la Exégesis, en la cual, el principio rector era "plenitud lógicamente necesaria en la legislación escrita". Durante este periodo la corriente dominante de la doctrina concluía de manera absoluta la no existencia de lagunas en la ley. El argumento irrefutable era pretexto del silencio, de la oscuridad o de la insuficiencia de la ley, podrá ser acusado como culpable de denegación de justicia.

Durante el curso del siglo XX esta argumentación simple resultó rápidamente insuficiente ante la profunda renovación de la teoría del Derecho. Pero, curiosamente, aun ante la evolución teórica, la conclusión siguió siendo la misma: las lagunas en el Derecho no existen.

En efecto, cualquiera que sea la premisa teórica por medio de la cual el problema resulte analizado, se constata de manera evidente la perfecta convergencia de los resultados. Por ejemplo, para un positivista normativista como Kelsen, el tema de las lagunas en el Derecho no puede ser tenido en cuenta para la validación de las normas, sino que aquéllas se derivan de una apreciación política de su contenido (1962, pp. 329 y ss.). En su criterio, por razones lógicas, no pueden existir vacíos en el sistema normativo (contra: P. Amselek, "a propósito de la teoría kelseniana de la ausencia de lagunas en el derecho", 1988, t. 33, p. 283). En cambio, para los iusnaturalistas lo que justifica esta solución no son razones lógicas, sino razones ligadas al contenido mismo del Derecho Natural. En fin, las perspectivas realistas contraponen la 
idea de que el juez tiene el poder de dar, con total libertad, la solución particular al caso que le ha sido asignado y, por consiguiente, el poder discrecional de subsanar las lagunas del Derecho.

En consecuencia, por razones diferentes, estas tres aproximaciones teóricas coinciden en admitir la ausencia de lagunas en el Derecho. ¿Será entonces necesario considerar que el tema de las lagunas no es en realidad más que un falso problema? Una respuesta afirmativa sería ciertamente apresurada, porque, de hecho, el tema de las lagunas es recurrente en el discurso jurídico. Los ejemplos no faltan, cuando vemos a los autores denunciar la existencia de vacíos jurídicos y desear que las autoridades normativas o jurisdiccionales subsanen las lagunas. Así, para detenernos en un ejemplo muy reciente, responsables políticos de primer plano y algunos autores han lamentado que la Constitución Francesa no consagre explícitamente principios como el de la dignidad de la persona humana, de la paridad en o entre las mujeres y los hombres o el de la diversidad. ¿Cómo resolver esta aparente paradoja entre la ausencia de lagunas en el Derecho y la constatación de su existencia por parte de autores o interpretes del Derecho? Parte de la explicación se puede adelantar: si fuera inexacto considerar que las lagunas existen en Derecho, como es posible constatar que lo actores o los intérpretes no han cesado de dar a conocer su presencia a fin de alcanzar que se subsanen dichas lagunas.

A partir de esta perspectiva serán considerados sucesivamente: en primer lugar, las lagunas puestas de presente por la interpretación del derecho constitucional (I) y en segundo lugar, el derecho constitucional, creado a partir de la interpretación de las lagunas (II).

\section{LAS LAGUNAS REVELADAS A TRAVÉS DE LA INTERPRETACIÓN DEL DERECHO CONSTITUCIONAL}

Cuando hacemos alusión a las lagunas reveladas por la interpretación del Derecho, nos limitamos a señalar que es simplemente el intérprete de la norma quien constata la existencia de las lagunas. Esta constatación bien entendida es significativa. Así, después de haber evidenciado las lagunas, parece indispensable hacer surgir las funciones que se derivan de esta verificación.

\section{Identificación de las lagunas}

Tratándose de la identificación de las lagunas, es necesario, ante todo, señalar que ésta se fundamenta en razones diferentes. Cuando el intérprete de una norma constata la existencia de una laguna, generalmente es porque hay ausencia total de norma en una materia determinada o porque la norma existente es insuficiente.

La ausencia total de norma se parece a lo que comúnmente Ilamamos un vacío jurídico. Generalmente, éste resulta de la aparición de un problema, hasta ese momento inédito, puesto a consideración del intérprete de la norma. El vacío jurídico puede resultar igualmente de la nueva comprensión de un asunto ya abordado en el pasado, pero regulado esta vez de manera diferente. Uno de los mejores ejemplos en Derecho Constitucional francés reside, sin duda, en el principio de la seguridad jurídica que es generalmente descrito como "faltante-ausente" en el texto de la Constitución francesa.

En cuanto a la insuficiencia de la norma de Derecho, ella puede resultar de la generalidad de los términos empleados. Es, entonces, la generalidad, por su imprecisión la cual aumenta, en la medida en que se asciende en la jerarquía de normas. Es la consecuencia lógica de la teoría de la formación 
de derecho por grados. Al respecto, podemos recordar la expresión propuesta por Bonnard, seguido de Merkl, según la cual, mientras la norma primaria sea más abstracta, el autor encargado de elaborar la norma secundaria tendrá más libertad de acción (RDP, 1928, p. 668 et al.). Aquí entonces los ejemplos abundan, en particular, si focalizamos la atención en ciertos estándares o "nociones de contenido variable", como por ejemplo, el principio de igualdad o recientemente esa noción forzosamente ambigua de la "identidad constitucional de Francia" que el Consejo Constitucional ha "descubierto" luego de una decisión del 30 de noviembre de 2006, proferida a propósito de una ley relativa al sector de la energía (Rec, p. 120).

En consecuencia, a la pluralidad de razones para que existan lagunas corresponde una diversidad de tipos de lagunas. Para Kelsen el punto de partida para el paso a las lagunas auténticas que recordemos desde su perspectiva normativista, no puede existir y, por otra parte, las simples lagunas técnicas, es decir, aquéllas que resultan de una omisión de la autoridad normativa, según sus propios términos resultan de "una discordancia entre el derecho positivo y el derecho deseado".

Por su parte, Chaim Perelman sugirió una tipología tripartita de lagunas, distinguiendo, en primer lugar, las lagunas intra legem que son las lagunas propias de construcción de la norma. Éstas remiten más o menos a lo que Kelsen denominó bajo el término de laguna técnica. Posteriormente, identifica lo que denomina las lagunas preter legem y contra legem. Según él, se trata dentro de estas dos hipótesis de lagunas, del resultado de la interpretación; señalemos que por razones diferentes Kelsen y Perelman (1984) consideran que sólo las lagunas intra legem son, propiamente hablando, lagunas. Sobre este punto existe entonces una concordancia de acercamientos teóricos que hubiéramos podido creer a priori divergentes. Estos dos autores recogen de esta manera la definición implícita contenida en el artículo 4 del Código Civil
Las lagunas pueden resultar del silencio de la insuficiencia o de la oscuridad de los textos. Esto conduce a unirse a la distinción, quizás la más simple que ya fue esbozada, entre las lagunas referidas a la existencia de un vacío jurídico y las lagunas referidas a la insuficiencia de la norma. El criterio de distinción reside en el grado de intensidad variable de esas lagunas.

La tipología de las lagunas es en sí misma significativa. Ésta contiene un inicio acerca del orden normativo. Ahora bien, es necesario interrogarse sobre las funciones derivadas de la puesta en evidencia de las lagunas.

\section{Las funciones de la puesta en evidencia de las lagunas}

Éstas son principalmente de dos clases. En primer lugar, la función aparente sería denunciar la inseguridad jurídica, creada por el vacío jurídico o por la insuficiencia de la norma. En efecto, esta idea se impone progresivamente, incluso en Francia, para la cual la seguridad jurídica constituye un pilar del Estado de Derecho. Roubier hablaba a este propósito de su "valor esencial" para el sistema jurídico. La seguridad jurídica es al menos el conocimiento a priori de las reglas generales que se pueden aplicar en un caso particular. Generalmente, se entiende por ello la previsibilidad de las soluciones dadas por los intérpretes de la norma. En este sentido, las lagunas aparecen como fallas en la coherencia general del sistema. En este caso, ellas ponen en peligro la seguridad jurídica.

En segundo lugar, debemos resaltar una función subyacente. Encubierta por un discurso descriptivo que denuncia los riesgos de la inseguridad jurídica generada por las lagunas, se transparenta un discurso prescriptito de los intérpretes. La invocación de las lagunas en el Derecho también tiene un valor argumentativo, un valor retórico. El objeto de esta invocación está destinado esencialmente a no aplicar el Derecho existente, sino un Derecho propuesto o creado por el intérprete. Lo anterior 
tiene el efecto, no solamente de legitimar su intervención, sino también aumentar el alcance de su poder discrecional. Sin embargo, este aumento del poder discrecional del intérprete es una medalla que tiene una contra cara en la medida en que conduce a una serie de inconvenientes.

Más allá de crearse un posible "gobierno de los jueces", los riesgos de eventuales arbitrariedades no se pueden descartar. Para contra balancear este argumento, es necesario, sin duda, hacer alusión al espíritu de las reflexiones de Diderot en sus Observaciones sur le Nakaz: "es mucho más importante tener buenos jueces que buenas leyes. Las mejores leyes son vanas con un mal juez y las peores leyes pueden ser corregidas por buenos jueces". Además, una ventaja con despreciable del descubrimiento de las lagunas por el intérprete es que éstas permiten esbozar soluciones futuras.

En resumen, el poder discrecional del intérprete consiste en una apertura del campo de las posibilidades. El descubrimiento de las lagunas implica un derecho de proporción, el cual puede conducir a una verdadera creación normativa. Debe, entonces, ser abordado la segunda línea de la argumentación, a saber: el Derecho Constitucional creado a partir de la interpretación de las lagunas.

\section{EL DERECHO CONSTITUCIONAL CREADO A PARTIR DE LA INTERPRETACIÓN DE LAS LAGUNAS}

Decir que existe un derecho creado a partir de la interpretación de las lagunas, supone decir que el intérprete se sitúa igualmente al final de la cadena normativa. La creación del Derecho por el intérprete es el resultado buscado, luego del descubrimiento de las lagunas (Melin-Soucramanien, 2005). Podemos establecer una distinción entre los resultados de la interpretación: por una parte, los resultados inmediatosy, por otra parte los resultados mediatos de la interpretación.

\section{Los resultados inmediatos de la interpretación}

Los resultados inmediatos de la interpretación consisten en subsanar las lagunas en el Derecho; estas lagunas pueden ser subsanadas directamente por el juez, o por los mismos textos. Cuando se trata de subsanar lagunas por parte del juez, dos modalidades son posibles en función del grado de intensidad de la laguna y del grado del poder discrecional de la autoridad jurisdiccional.

La primera hipótesis es aquélla en la cual el juez tiene un poder supletorio, porque existe un vacío jurídico. Entonces, su poder de creación del Derecho es elevado al máximo. En el Derecho Constitucional francés, el mejor ejemplo está, sin duda, representado por la creación de los principios fundamentales reconocidos por las leyes de la República por iniciativa generalmente del Consejo Constitucional y excepcionalmente del Consejo de Estado.

La segunda hipótesis es aquélla en la cual el juez no tiene un poder supletorio, sino solamente de precisión, de extensión del campo de aplicación de la norma. El método de interpretación es entonces esencialmente el del razonamiento por analogía, es decir, se supone que existe ya una norma que el juez va a aplicar a casos análogos.

Así, por ejemplo, en la Constitución Italiana no existe la disposición que garantice expresamente el derecho al medio ambiente. Pero, en cambio, su artículo 32 consagra el derecho a la protección de la salud como un derecho fundamental. Por analogía, la Corte Constitucional italiana, en una serie de sentencias proferidas a partir de 1988, reconoció la existencia de un derecho fundamental al medio ambiente por conexidad en el derecho a la salud. En Francia, como lo sabemos se ha seguido otra vía, "incorporando" a al Constitución una Carta del Medio Ambiente en 2005. 
Pero, lo más frecuente es que cuando se subsanan las lagunas, ello haya sido provocado por la verificación, en un nivel más alto, de la existencia de un vacío jurídico. Esta verificación corresponde claramente al juez. Así el juez constitucional, en 1974, en una decisión relativa a la declaración de vacancia por el presidente Pompidou, el Consejo Constitucional señaló una laguna relativa al deceso $\mathrm{y}$ al impedimento de un candidato en el curso del proceso de elección presidencial. Esta decisión obligó en 1976 a la revisión del artículo $7^{\circ}$ de la Constitución. Más allá de los resultados inmediatos de la interpretación de las lagunas, vemos que aparecen resultados mediatos, o a más largo tiempo.

\section{Los resultados mediatos de la interpretación}

Los resultados mediatos de la interpretación para subsanar lagunas conciernen a los efectos en el orden jurídico considerado en su conjunto. Así, subsanar las lagunas puede, con el propósito de asegurar la completud o la perfección del sistema normativo, conducir a una rigidez excesiva del Derecho. En efecto, la ilusión de poder crear un orden jurídico perfecto a través de la ocupación de interticios abiertos en las normas del Derecho Positivo puede crear en realidad un cierre.

Por ejemplo, en Alemania en 1990 el legislador dio prueba de su audacia afirmando que "desde la fecundación comienza una nueva vida humana". Esta posición contrasta fuertemente con la situación francesa evidentemente, pero también con la italiana o con la española.
Desde este punto de vista, las lagunas contribuyen a crear un "Derecho flexible" para retomar una expresión ya consagrada. Igualmente, si este "impresionismo" jurídico es mantenido permanentemente de manera sabia. En este ámbito, las lagunas en el Derecho conservan la indeterminación y, por lo mismo, la reserva de la autoridad normativa que garantice la no confusión entre las esferas respectivas del Derecho y la moral.

Por ejemplo, estos mismos aspectos se podrían aplicar al caso del encuadramiento jurídico de la clonación. De hecho, en ese caso preciso, la otra dimensión del problema tiene que ver con la ausencia de una definición científicamente segura de la clonación. Llenar esta pretendida laguna, reviviría evidentemente la cuestión de la oportunidad de la adecuación del Derecho a los hechos.

El mismo argumento vale, a fortiori, puesto que se trata de las propuestas actuales con miras a reformar el preámbulo de la Constitución, a fin de incorporar conceptos tales como el de la diversidad, la dignidad o la paridad. Aquí es necesario subrayar que ciertas lagunas deben, sin duda, subsistir en un texto constitucional, en el sentido que es verdad que una Constitución gana, según las palabras prestadas de Napoleón Bonaparte, al ser al mismo tiempo "breve y obscura". 